\title{
BMJ
}

\section{Association of change in daily step count over five years with insulin sensitivity and adiposity: population based cohort study}

\author{
T Dwyer, director, ${ }^{1,2}$ A-L Ponsonby, group leader, ${ }^{1,2}$ O C Ukoumunne, senior research fellow, ${ }^{1}$ A Pezic, research \\ assistant, ${ }^{1}$ A Venn, deputy director, ${ }^{2}$ D Dunstan, research fellow, ${ }^{3}$ E Barr, epidemiologist, ${ }^{3}$ S Blair, professor, ${ }^{4}$ J \\ Cochrane, data manager, ${ }^{2}$ P Zimmet, professor, ${ }^{3}$ J Shaw, associate director ${ }^{3}$
}

${ }^{1}$ Murdoch Children's Research

Institute, Royal Children's Hospital, Parkville, Melbourne 3052, Australia

${ }^{2}$ Menzies Research Institute, University of Tasmania, Hobart 7000, Australia

${ }^{3}$ Baker IDI Heart and Diabetes Institute, Melbourne 3004, Australia

${ }^{4}$ Arnold School of Public Health, University of South Carolina, Columbia, SC 29208, USA Correspondence to: T Dwyer terry.dwyer@mcri.edu.au

Cite this as: $B M J$ 2010;341:c7249 doi:10.1136/bmi.c7249

\section{ABSTRACT}

Objectives To investigate the association between change in daily step count and both adiposity and insulin sensitivity and the extent to which the association between change in daily step count and insulin sensitivity may be mediated by adiposity.

Design Population based cohort study.

Setting Tasmania, Australia.

Participants 592 adults (men ( $n=267$ ), mean age 51.4 (SD 12.2) years; women ( $n=325)$, mean age 50.3 (12.3) years) who participated in the Tasmanian component of the national AusDiab Study in 2000 and 2005.

Main outcome measures Body mass index, waist to hip ratio, and HOMA insulin sensitivity at follow-up in 2005.

Results Over the five year period, the daily step count decreased for $65 \%(n=382)$ of participants. Having a higher daily step count in 2005 than in 2000 was independently associated with lower body mass index (0.08 (95\% confidence interval 0.04 to 0.12 ) lower per 1000 steps), lower waist to hip ratio (0.15 (0.07 to 0.23 ) lower), and greater insulin sensitivity (1.38 (0.14 to 2.63) HOMA units higher) in 2005. The mean increase in HOMA units fell to 0.34 (-0.79 to 1.47 ) after adjustment for body mass index in 2005.

Conclusions Among community dwelling, middle aged adults, a higher daily step count at five year follow-up than at baseline was associated with better insulin sensitivity. This effect seems to be largely mediated through lower adiposity.

\section{INTRODUCTION}

Recent increases in type 2 diabetes and obesity in many countries, including the United States and Australia, have been partly attributed to declines in physical activity. ${ }^{1}$ Randomised controlled trials have shown that interventions involving physical activity reduce body mass index and the progression to insulin resistance, but most were done in selected groups, such as people with impaired glucose tolerance, or had composite interventions including diet as well as physical activity. ${ }^{2-5}$
Data from cohort studies relying on measurement of physical activity by questionnaires strongly support an independent role for physical activity. In a review of 10 cohort studies with follow-up ranging from 5 to 17 years, self reported regular physical activity of moderate intensity, compared with being inactive, was associated with a reduced risk of type 2 diabetes (pooled relative risk 0.69, 95\% confidence interval 0.58 to 0.83 ). Some of this association seemed to be mediated through a beneficial effect of activity on body mass index (body mass index adjusted pooled relative risk $0.83,0.76$ to 0.90$){ }^{6}$

The advent of objective measures of physical activity has enabled more precise measurement of activity patterns, thus minimising misclassification on this exposure. ${ }^{7}$ Theoretically, this should lead to better estimates of the association of physical activity with outcomes of interest. However, the randomised trials and cohort studies in which objective measures of physical activity have been incorporated have failed to clearly identify an effect on insulin sensitivity, despite evidence for reduced body fatness. A systematic review of eight randomised trials carried out before 2007 found that interventions based on use of a pedometer, ${ }^{8}$ despite achieving a mean increase of 2183 steps per day, failed to produce any effects on fasting plasma glucose. Similarly, in cohort studies, use of a pedometer was associated with a 2490 step increase but with no change in plasma glucose. ${ }^{8}$ Both study types were of short duration; the trials had a median duration of 12 weeks, and the median for the cohort studies was nine weeks. More recently, two larger cohort studies involving 192 and 367 participants, and objective measurement of physical activity, have reported conflicting findings about the association of moderate and vigorous activity, and sedentary time, with insulin resistance..$^{910}$

The aim of this study was to examine the relation between change in physical activity (measured objectively by pedometer and also by self reported questionnaire measures) over a five year period and each of body mass index, waist to hip ratio, and insulin sensitivity (as 
estimated by the HOMA insulin sensitivity index) at the end of the five years, in a large population based sample of participants from the AusDiab study. ${ }^{11}$

\section{METHODS}

\section{Participants}

The AusDiab study estimated the national prevalence of diabetes and related risk factors in 2000. Participants came from a national sample of adults aged 25 years and older from six states and the Northern Territory in 2000. Methods and central findings from this large cross sectional survey have been reported elsewhere. ${ }^{11}{ }^{12}$ In Tasmania, six census collection districts were randomly sampled from the Tasmanian list, based on the 1996 Australian census. Within these districts, adults aged 25 years and over were invited to participate, and $40 \%$ of those who completed a household questionnaire participated in the full survey $(\mathrm{n}=$ 1848). ${ }^{11}$ We excluded from this study 175 participants who had diabetes mellitus in 2000 , as assessed by an oral glucose tolerance test. Of the remaining 1611 who had a result from an oral glucose tolerance test in 2000 , we included the 592 participants who provided pedometer data for two days in both 2000 and 2005 in the analyses reported in this paper. ${ }^{7}$ All participants gave their consent by signing a consent form.

\section{Study measures}

In both 2000 and 2005 the participants were invited to attend a clinic, where anthropometric and other measurements were collected as described below and a venous blood sample was obtained. Instructions for the use and return of the pedometer were provided.

Questionnaire assessment of recent physical activity time In 2000 and 2005 participants used the active Australia questionnaire to report the frequency and duration of physical activity in the previous week. ${ }^{13}$ Physical activity was estimated from the time spent walking for recreation or transport and doing non-domestic activity and the time spent doing moderate intensity and vigorous non-work activity during the previous week. These questions have been found to provide reliable and valid estimates of adults' physical activity. ${ }^{14} \mathrm{We}$ calculated a weighted sum of the responses (with vigorous activity given double weighting) to quantify the total hours of physical activity time. ${ }^{7}$

\section{Objective assessment of physical activity with pedometer} The pedometer protocol has been previously described. ${ }^{7}$ The pedometers used were the Omron HJ-003 and Omron HJ-102, with regular checks for accuracy of counts. Research staff demonstrated the use, placement, and operation of the pedometer, and the participants were then given printed instructions and a diary to record daily steps. ${ }^{7}$ The summary measure used was the average daily number of steps over two consecutive days. A previous study had reported intraclass correlations (to quantify agreement) of 0.71-0.84 for any two days of recording, and two consecutive days captured $89 \%$ of the variance of a seven day recording period. ${ }^{15}$

\section{Diet and other lifestyle and demographic attributes}

Current smoking status and level of education were determined from the questionnaire. Usual dietary intake over the previous 12 months was assessed with a self administered validated food frequency questionnaire developed by the Anti-Cancer Council of Victoria. ${ }^{11}$ We calculated nutrient intake by multiplying the frequency of food consumption by standard portion weights to obtain daily food weights. ${ }^{16} \mathrm{We}$ then converted these to nutrient intakes based on the NUTTAB 95 nutrient composition data. ${ }^{16}$ Alcohol intake also came from the food frequency questionnaire and was incorporated into the calculation of total energy intake (kJ/day). ${ }^{11}$ We classified higher education as participants having completed a university or technical college degree, and socioeconomic status was measured by using thirds derived from the Australian based socioeconomic indexes for area. ${ }^{17}$

\section{Clinical examination}

Height was measured to the nearest $0.5 \mathrm{~cm}$ without shoes, and weight was recorded to the nearest $0.1 \mathrm{~kg}$. Waist circumference was measured half way between the lower border of the ribs and the iliac crest on the horizontal plane. Hip circumference was measured at the widest point over the buttocks. For each of waist and hip circumference, the average of two consecutive measurements within $2 \mathrm{~cm}$ was recorded. Body mass index was calculated as weight $(\mathrm{kg}) /$ height $(\mathrm{m})^{2}$. Participants were classified as normal weight (body mass index $<25)$, overweight $(25-<30)$, or obese $(\geq 30)$. We used waist to hip ratio and body mass index as measures of adiposity.

\section{Assessment of HOMA insulin sensitivity}

Venous blood was collected after an overnight fast (eight hours). Blood specimens were centrifuged on site and transported daily to the central laboratory. Serum samples for insulin were stored at $-80^{\circ} \mathrm{C}$ until assayed. In 2000 plasma glucose levels were determined by using an Olympus AU600 automated analyser. Serum insulin was measured by using a human insulin specific radioimmunoassay kit (Linco Research, St Charles, MO), only for participants aged over 35 in $2000(\mathrm{n}=527) .{ }^{18}$ In 2005 fasting plasma glucose was measured by using a Roche Modular (Roche Diagnostics, Indianapolis, IN, USA) with a spectrophotometric hexokinase method, ${ }^{18}$ and the laboratory analysis methods in 2000 and 2005 were comparable. ${ }^{19}$ In 2005 serum insulin was measured by using a chemiluminescence method for all participants. The homoeostatic model assessment of insulin sensitivity (HOMA insulin sensitivity) was calculated by the HOMA2 program. ${ }^{20}$ The HOMA model is the most widely used surrogate marker for assessing insulin sensitivity and $\beta$ cell function in clinical and epidemiological studies. ${ }^{21}$ 


\section{Statistical analysis}

We used Stata version 10.1 for all analyses. We use numbers and percentages to describe categorical variables and means and standard deviations or medians and interquartile ranges to describe quantitative variables. We used linear regression to describe the relation of each of the three outcomes (body mass index, waist to hip circumference, and HOMA insulin sensitivity) in 2005 with change between 2000 and 2005 in each of pedometer measured and self reported physical activity measures in separate models (that is, six separate models). For example, we used change in pedometer measured activity (step count) as a continuous predictor, adjusting for the corresponding pedometer measured activity score (step count) in 2000, also in continuous form. In addition to these "unadjusted" analyses, we incorporated the potential baseline (2000) confounders of age, sex, body mass index, waist to hip ratio, HOMA insulin sensitivity, socioeconomic status, total energy from all sources $(\mathrm{kJ})$, current smoking status, alcohol consumption (g/day), education, and length of follow-up into adjusted models. In addition, because insulin was measured slightly differently in 2005 and 2000, we did a supplementary analysis in which we analysed the ranks of the insulin sensitivity scores in 2000 and 2005 in the adjusted model rather than the actual values.

We tested modification of the effects of change in pedometer measured and self reported physical activity on all three outcomes, examining sex and family history of diabetes in 2000 as potential effect modifiers. For these analyses, we included product terms for the interaction between change in activity and each of the potential effect modifiers in regression models of the outcomes. We also investigated whether effects of pedometer measured physical activity on HOMA insulin sensitivity were mediated through change in body anthropometry, by adding body mass index in 2005 to the adjusted models and observing the resulting change in estimates. In all models, we checked that continuous covariates had linear relations with the outcomes, by using the method of fractional poly nomials. ${ }^{22}$ We used normal probability plots to confirm that the residuals had a symmetrical distribution and plots of the residuals against predicted values to confirm an absence of marked deviation from constant variance. As a sensitivity analysis, we fitted population weighted models to determine whether the results were influenced by differences in demographic characteristics between the 592 participants analysed in this study and the remaining participants from the AusDiab cohort. We used weights that adjust the analysed sample to be representative of the full AusDiab cohort with respect to sex, age, and socioeconomic status in 2000. The weights were the inverse of the probability of being analysed in this study, and we calculated them by using the probabilities obtained after we fitted a logistic regression of whether analysed in this study $(0=$ not analysed; $1=$ analysed $)$ on sex, age, and socioeconomic status, using the full AusDiab cohort. Little and Rubin have referred to this approach to handling missing data of weighting the participants who provide complete data as propensity weighting. ${ }^{23}$

We also examined the relation between pedometer measured physical activity and the study outcomes by using a categorical ordinal variable that summarises the profile of change in objective physical activity. We created the variable as follows. In both 2000 and 2005 we divided the study participants, by using tertile defined cut-off points of the daily steps, into groups. We classified participants who were in the lowest step category in both 2000 and 2005 as persistent low steps (lowest third at both waves) and those who dropped by one or two categories between 2000 and 2005 as decreasing steps (decline of one or two categories). The other groups were persistent moderate steps (middle third at both waves), increasing steps (increase of one or two categories), and persistent high steps (highest third at both waves). We tested for evidence of a difference in means across the five groups. We report the adjusted difference in the mean outcomes at 2005 between each category of step change and the persistent low steps (reference) category.

\section{RESULTS}

Table 1 shows the characteristics of the participants. The cohort observation period ranged from 4.8 to 5.0 years, with a mean follow-up of 4.9 (SD 0.03) years. In 2000

\begin{tabular}{|c|c|c|c|c|}
\hline \multirow[b]{2}{*}{ Characteristic } & \multicolumn{2}{|c|}{ Baseline (2000) } & \multicolumn{2}{|c|}{ Follow-up (2005) } \\
\hline & Men & Women & Men & Women \\
\hline Age (years) & $51.4(12.2)$ & $50.3(12.3)$ & $56.3(12.2)$ & $55.3(12.3)$ \\
\hline Median (interquartile range) physical activity, by pedometer (steps/day) & $10172(7435-13928)$ & $10969(7889-14$ 402) & $9108(6261-11792)$ & $8700(5843-11963)$ \\
\hline $\begin{array}{l}\text { Median (interquartile range) physical activity in previous week, by questionnaire } \\
\text { (hours) }\end{array}$ & $4.5(2-9.5)$ & $2.8(0.8-6.5)$ & $5.6(2-11)$ & $3.5(1-8)$ \\
\hline Waist circumference $(\mathrm{cm})$ & $95.5(10.0)$ & $82.5(10.9)$ & $98.3(10.1)$ & $85.6(11.3)$ \\
\hline Waist to hip ratio & $0.94(0.07)$ & $0.81(0.07)$ & $0.94(0.06)$ & $0.81(0.06)$ \\
\hline Weight (kg) & $83.8(12.0)$ & $68.3(12.0)$ & $85.1(13.2)$ & $69.9(13.3)$ \\
\hline Height $(\mathrm{cm})$ & $175.8(6.7)$ & $162.2(6.0)$ & $174.8(6.8)$ & $161.0(6.1)$ \\
\hline Body mass index $\left(\mathrm{kg} / \mathrm{m}^{2}\right)$ & $27.1(3.4)$ & $26.0(4.2)$ & $27.8(3.7)$ & $26.9(4.7)$ \\
\hline Median (interquartile range) alcohol consumption (g/day) & $12(3-28)$ & $2(0-11)$ & $13(3-28)$ & $3(0-11)$ \\
\hline Percentage (No) smokers & $14.8(39 / 263)$ & $11.8(38 / 322)$ & $11.2(29 / 259)$ & $10.6(33 / 311)$ \\
\hline
\end{tabular}


Table 2 Changes in daily steps activity over time $(n=592)$

\begin{tabular}{lc}
$\begin{array}{l}\text { Change in activity } \\
\text { Average steps/day, 2000-5 }\end{array}$ & $\%$ (No) participants in each category \\
\hline Decrease $>3000$ & $37.2(220)$ \\
\hline Decrease 1000-3000 & $18.8(111)$ \\
\hline Decrease 1-1000 & $8.6(51)$ \\
\hline Increase 0-1000 & $8.3(49)$ \\
\hline Increase 1000-3000 & $14.0(83)$ \\
\hline Increase 3000 & $13.2(78)$ \\
\hline Step change category* & $18.4(109)$ \\
\hline Persistent low steps & $26.5(157)$ \\
\hline Decreasing steps & $12.0(71)$ \\
\hline Persistent moderate steps & $26.4(156)$ \\
\hline Increasing steps & $16.7(99)$ \\
\hline Persistent high steps
\end{tabular}

*Persistent low steps=lowest third at both waves; decreasing steps=decline of 1 or 2 thirds; persistent moderate steps=middle third at both waves; increasing steps=increase of 1 or 2 thirds; persistent high steps=lowest third at both waves.

the mean age was $51.4(12.2)$ years for men and 50.3 (12.3) for women. A high proportion of participants were overweight or obese in 2000 (men: 57.4\% (152/ $265)$ overweight and 17.7\% (47/265) obese; women: $36.9 \%(120 / 325)$ overweight and $16.0 \% \quad(52 / 325)$ obese). The mean body mass index was 27.1 (3.4) for men and 26.0 (4.2) for women. A further increase in weight occurred over the five year period, as well as a decline in daily step activity. Table 2 shows that although most participants had a decline in steps over time, more than a third had more daily steps by 2005 , and $16.7 \%$ stayed in the persistent high steps category.

We compared participants analysed here $(n=592)$ with the AusDiab participants in Tasmania without diabetes in 2000 who were not analysed $(n=1050)$. The two groups were similar in age (mean 50.8 (SD 12.3) v $50.5(15.2))$, proportion of men $(45.1 \% v$ $45.3 \%$ ), weight (mean $75.3(14.2) \mathrm{kg} v 76.3(16.3) \mathrm{kg}$ ), and height (mean $168.3(9.3) \mathrm{cm} v 168.3(9.5) \mathrm{cm})$. The proportion of obese participants was slightly lower among those analysed in this study (16.8\% v 20.9\%).

In 2000 a positive association existed between average daily step count and self report of walking/moderate activity (Pearson's $r=0.21 ; \mathrm{P}<0.001$ ) and also a weaker association with self reported vigorous activity $(r=0.13 ; \mathrm{P}=0.001)$. The corresponding correlations with average daily step count in 2005 were $r=0.22$ $(\mathrm{P}<0.001)$ for walking/moderate activity and $r=0.12$
$(\mathrm{P}=0.005)$ for vigorous activity. In 2005 the mean HOMA insulin sensitivity was 108.0 (62.6) units for men and 120.4 (61.8) units for women.

Relation between physical activity and outcomes in 2005 The fractional polynomials analysis showed that the variables measuring activity in 2000 and change in activity had linear relations with the three study outcomes in 2005. We found generally strong evidence, in the unadjusted and adjusted analyses, that both higher daily steps in 2000 and higher daily steps in 2005 than in 2000 were associated with a lower body mass index (table 3), a lower waist to hip ratio (table 4), and greater insulin sensitivity in 2005 (table 5) when we considered both simultaneously in a joint prediction model. The tables show the adjusted results. In the supplementary adjusted analysis in which we examined the ranked insulin sensitivity scores, higher daily steps in 2000 and higher daily steps in 2005 than in 2000 were associated with greater HOMA insulin sensitivity in 2005 (both $\mathrm{P}=0.03$ ). Reported physical activity time was also generally associated with the outcomes. In addition, higher reported physical activity in 2000 and higher physical activity in 2005 than in 2000 were associated with a lower body mass index and greater insulin sensitivity in 2005 when both were considered simultaneously in a joint prediction model.

The estimates, if they correspond to causal effects, would imply the following quantitative results. If a relatively inactive person increased his or her daily steps by 10000 a day, ${ }^{24}$ the resultant change would be a decrease in body mass index of 0.83 units. The resultant 13.85 unit improvement in HOMA insulin sensitivity represents a $12.8 \%$ increase from the population mean values for men and an $11.5 \%$ increase for women. For a relatively inactive person increasing the number of steps by 2000 a day (similar to a previous recommendation of 3000 steps a day for five days ${ }^{25}$ ), the resultant changes would be a decrease of 0.16 units for body mass index and an improvement of 2.76 units for HOMA insulin sensitivity, after adjustment for the factors listed in the footnote of table 3 .

Tests of interaction showed little evidence that the effects of the physical activity measures are modified by sex and family history of diabetes (data not shown). In addition, the effect of a change in steps on body mass index, waist to hip ratio, and HOMA insulin sensitivity in 2005 did not vary according to average daily steps in 2000 .

Table $3 \mid$ Association between indicators of physical activity level at baseline, change in levels between 2000 and 2005, and body mass index in 2005

\begin{tabular}{|c|c|c|}
\hline Exposures used in separate models & Mean change* $(95 \% \mathrm{Cl})$ & $P$ value \\
\hline Average daily steps in 2000 , by pedometer (per 1000 higher per day) & $-0.06(-0.10$ to -0.02$)$ & 0.01 \\
\hline Change in average daily steps $2000-5$, by pedometer (per 1000 higher per day) & $-0.08(-0.12$ to -0.04$)$ & $<0.001$ \\
\hline Total physical activity time in 2000 , by questionnaire (per 1 hour higher per week) & $-0.04(-0.07$ to -0.004$)$ & 0.03 \\
\hline Change in total physical activity time $2000-5$, by questionnaire (per 1 hour higher per week) & $-0.07(-0.10$ to -0.04$)$ & $<0.001$ \\
\hline
\end{tabular}


Table $4 \mid$ Association between indicators of physical activity level at baseline, change in levels between 2000 and 2005 , and waist to hip ratio in 2005

\begin{tabular}{llc}
\hline Exposures used in separate models & Mean change* $(95 \% \mathrm{Cl})$ & $-0.13(-0.21$ to -0.05$)$ \\
Average daily steps in 2000, by pedometer (per 1000 higher per day) & $-0.15(-0.23$ to -0.07$)$ \\
\hline Change in average daily steps 2000-5, by pedometer (per 1000 higher per day) & $-0.05(-0.12$ to 0.02$)$ \\
\hline Total physical activity time in 2000, by questionnaire (per 1 hour higher per week) & $-0.09(-0.15$ to -0.04$)$ & 0.16 \\
\hline Change in total physical activity time 2000-5, by questionnaire (per 1 hour higher per week) & 0.002
\end{tabular}

*Adjusted for age, sex, waist to hip in 2000 , socioeconomic status in 2000 , total energy intake from all sources (kJ) in 2000, smoking status in 2000 , alcohol consumption (g/day) in 2000, education in 2000 , and length of cohort follow-up. Note that separate models were fitted for each type of activity (exposure).

The results of further analyses suggested that the apparent effect of higher step activity on enhanced HOMA insulin sensitivity was largely mediated through the change in adiposity; the adjusted regression coefficient for change in steps dropped from 1.38 to 0.34 (95\% confidence interval -0.79 to $1.47 ; \mathrm{P}=0.56)$ after adjustment for body mass index in 2005. The addition of body mass index in 2005 reduced the magnitude of the regression coefficient between change in steps and insulin sensitivity by $75 \%$.

When we weighted the regression analyses to allow for potential loss to follow-up, taking account of the composition of the entire group of AusDiab participants without diabetes in our region in 2000, the results were similar to those of the main analysis (data not shown). This suggests that no loss to follow-up bias exists related to lack of representativeness on the variables used to calculate the weights.

Relation of profile of change in steps between 2000 and 2005 with outcomes

To gain further insights into the effect of changing pedometer steps over time, we examined the association of the ordinal variable describing change in steps in relation to the study outcomes. The figure summarises the adjusted differences between the persistent low steps category (reference) and each of the other four categories with respect to mean outcome in 2005. Participants who maintained a persistently high step count between 2000 and 2005 had a mean difference of $-0.82(-1.39$ to -0.26$)$ body mass index units and 16.85 (0.13 to 33.57) units of HOMA insulin sensitivity in 2005 , relative to those who had persistently low step counts.

\section{DISCUSSION}

In this large population based cohort, a higher step activity at the end of a five year follow-up period than at the beginning was associated with a lower body mass index, lower waist to hip ratio, and higher HOMA insulin sensitivity at the five year follow-up. The observed association of step activity with enhanced HOMA insulin sensitivity was largely accounted for by body mass index in 2005 . This may indicate a mediation effect through change in body mass index. This finding is consistent with data from cohort studies that have used self reported measures of physical activity. ${ }^{6}$ This study enabled us to examine the association of the change in each of the pedometer steps and questionnaire measures of physical activity with obesity and insulin sensitivity, while adjusting for other lifestyle determinants such as diet, alcohol, and smoking.

\section{Comparison with other studies}

Participants who had a high daily step count at both 2000 and five years later had a lower body mass index ( 0.8 less) and higher HOMA insulin sensitivity (16.9 units higher) relative to those who had persistently low step count from 2000 to 2005 . Our estimate of the independent association of steps with body mass index $(0.16$ lower body mass index for an increase of 2000 steps) was lower than the 0.38 previously reported as the summary effect of pedometer use associated with a 2000-2500 change in steps. ${ }^{8}$ This may reflect the fact that many previous studies involved interventions to increase pedometer use, ${ }^{8}$ and other associated effects such as a greater awareness of energy balance and thus caloric restriction may have been present. We focused on a higher daily step activity, although most participants had a decline in step activity, to provide parallel results to those that would be available from interventions involving physical activity in this area.

\section{Strengths and limitations}

The major strengths of this study are its size, duration, and objective measure of physical activity and the

Table 5 The association between indicators of physical activity level at baseline, change in levels between 2000 and 2005 , and HOMA insulin sensitivity in 2005

\begin{tabular}{|c|c|c|}
\hline Exposures used in separate models & Mean change* $(95 \% \mathrm{Cl})$ & P value \\
\hline Average daily steps in 2000 , by pedometer (per 1000 higher per day) & $1.72(0.45$ to 2.99$)$ & 0.01 \\
\hline Change in average daily steps 2000-5, by pedometer (per 1000 higher per day) & $1.38(0.14$ to 2.63$)$ & 0.03 \\
\hline Total physical activity time in 2000 , by questionnaire (per 1 hour higher per week) & $1.35(0.31$ to 2.39$)$ & 0.01 \\
\hline Change in total physical activity time $2000-5$, by questionnaire (per 1 hour higher per week) & $1.27(0.42$ to 2.12$)$ & 0.004 \\
\hline
\end{tabular}



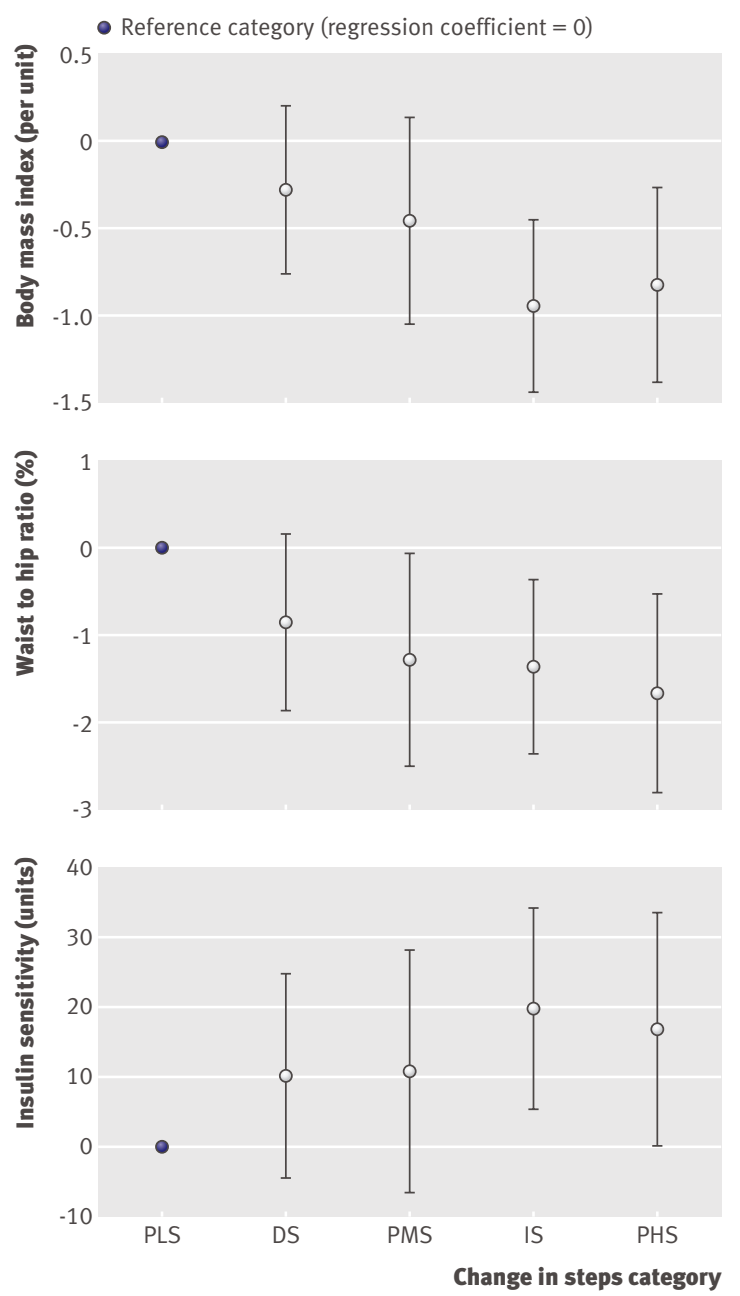

Adjusted mean difference in body mass index (BMI), waist to hip ratio (WHR), and HOMA insulin sensitivity in 2005 for each category of step change compared with persistent low steps category. Mean differences adjusted for age, sex, socioeconomic status in 2000 , total energy from all sources (kJ) in 2000 , smoking status in 2000 , alcohol consumption (g/day) in 2000 , education in 2000 , and length of cohort follow-up. BMI in 2005 outcome additionally adjusted for BM in 2000, WHR in 2005 outcome additionally adjusted for WHR in 2000, and HOMA insulin sensitivity in 2005 outcome additionally adjusted for HOMA insulin sensitivity in 2000 . PLS=persistent low steps (lowest third at both waves); DS=decreasing steps (decline of 1 or 2 categories);

PMS=persistent moderate steps (middle third at both waves); IS=increasing steps (increase of 1 or 2 categories);

PHS=persistent high steps (lowest third at both waves). Test of difference in mean outcomes across five groups: for BMI, $P=0.001$; for WHR, $P=0.03$; for insulin sensitivity, $P=0.09$

outcome measures. Pedometer assessments were made on two occasions spanning a five year interval in more than 500 people. In addition, at the same time points we measured physical activity by questionnaire and a range of other potential determinants of obesity.

In an observational study such as this, confounding is always a possible explanation for observed associations. We were able to control for baseline age, sex, body mass index, waist to hip ratio, HOMA insulin sensitivity, socioeconomic status, total energy intake from all sources $(\mathrm{kJ})$, current smoking status, alcohol consumption (g/day), education, and length of cohort follow-up. Adjustment for these factors did not materially alter the estimate of association. Loss to follow-up in a cohort study could also bias the results. We were able to account for this by weighting the results for the loss that occurred and found that this did not affect the estimate of the association between change in steps and insulin sensitivity. Further evidence supporting a possible causal connection is that these study results are supported by evidence from randomised trials, at least for body mass index, albeit over shorter timeframes and with smaller numbers.

As we collected data only in 2000 and 2005, we could not determine the exact timing of the change in number of steps taken or the duration of any change. We could only take the two measures as a summary estimate of what happened over the five years. Nevertheless, both steps in 2000 and change in steps between 2000 and 2005 were associated with a lower body mass index, a lower waist to hip ratio, and greater insulin sensitivity in 2005 .

The pedometer measures movement only in the vertical plane and therefore most readily captures walking or running type movements. It would miss activity in the horizontal plane, such as cycling, but probably captures most of the activity undertaken by adults of this age. Supporting this, it has been shown to be highly associated with accelerometry, a more complex measure of total body movement. The correlation coefficient relating the two measures has been estimated at 0.74 to $0.86 .^{26}$

We found only a modest association between the objective and reported measures of physical activity. This emphasises the importance of not relying on self report alone because of potential non-differential measurement error and reporting bias.

A guideline of 10000 steps a day is approximately equivalent to 5 miles $(8 \mathrm{~km})$ covered on foot, ${ }^{27}$ but the actual energy expenditure will vary depending on age, sex, height, leg length, and gait. ${ }^{28}$ An average woman's step length is $67 \mathrm{~cm}$ and that for a man is $76 \mathrm{~cm},{ }^{29}$ meaning that a woman walks a kilometre in 1493 steps and a man in 1316 steps. If the energy equivalent of walking $1 \mathrm{~km}$, based on the person's body weight, is approximately $200 \mathrm{~kJ}$ of energy, ${ }^{30}$ then an increase of 2000 steps would equate to an added energy expenditure of approximately $268 \mathrm{~kJ}$ for women and $304 \mathrm{~kJ}$ for men.

\section{Conclusions and policy implications}

These findings, confirming an independent beneficial role of higher daily step count on body mass index, waist to hip ratio, and insulin sensitivity, provide further support to promote higher physical activity levels among middle aged adults. The use of a pedometer for measuring physical activity allows quantification of the magnitude of these effects, in terms of daily step activity, which should assist future public health policy.

Our findings indicate that the relation between a change in steps over time and the relevant outcomes 


\section{WHAT IS ALREADY KNOWN ON THIS TOPIC}

Recent increases in type 2 diabetes and obesity have been partly attributed to declines in physical activity

Randomised controlled trials have shown that physical activity reduces body mass index and progression to insulin resistance, but most have been in selected groups or used composite interventions

Cohort study data relying on questionnaire measurement of physical activity support an independent role for physical activity in maintaining insulin sensitivity

\section{WHAT THIS STUDY ADDS}

Higher step activity in 2005 than in 2000 was associated with a lower body mass index, lower waist to hip ratio, and better insulin sensitivity in 2005, independent of dietary energy intake

The apparent beneficial effect of higher physical activity on insulin sensitivity seemed to be largely mediated by reduced adiposity

The association of change in daily step count over five years (independent of energy intake) with body mass index, waist to hip ratio, and insulin sensitivity has been quantified

is linear in nature. On this basis, we estimate that, in our setting, a sedentary person who takes a very low number of daily steps but who was able to change behaviour over five years to meet the popular 10000 daily step guideline would have a threefold improvement in HOMA insulin sensitivity compared with a similar person who increased his or her steps to meet the more recent recommendation of 3000 steps for five days a week. ${ }^{2425}$

In conclusion, among community dwelling people, an increase in physical activity over time, measured objectively by pedometer, is associated with better HOMA insulin sensitivity. This apparent beneficial effect seems to be largely mediated through a change in adiposity.

We thank the AusDiab study participants and all staff involved in the fieldwork of this study.

Contributors: TD, AV, IS, DD, EB, SB and PZ contributed to the conception and design of the study. TD, AV, EB, and JC contributed to the conduct of the study. TD, A-LP, OCU, AP, and JC contributed to the analysis and interpretation of data. TD, A-LP, OCU, and AP contributed to drafting the article. All authors contributed to revising the article critically for important intellectual content and approved the final version. TD the is guarantor.

Funding: The study received financial support from the Commonwealth Department of Health and Aged Care, Abbott Australasia, Alphapharm, Aventis Pharmaceutical, AstraZeneca, Bristol-Myers Squibb Pharmaceuticals, Eli Lilly (Australia), GlaxoSmithKline, Janssen-Cilag (Australia), Merck Lipha, Merck Sharp \& Dohme (Australia), Novartis Pharmaceutical (Australia), Novo Nordisk Pharmaceutical, Pharmacia and Upjohn, Pfizer, Roche Diagnostics, Sanofi Synthelabo (Australia), Servier Laboratories (Australia), BioRad Laboratories, HITECH Pathology, the Australian Kidney Foundation, Diabetes Australia, Diabetes Australia (Northern Territory), Queensland Health, South Australian Department of Human Services, Tasmanian Department of Health and Human Services, Territory Health Services, Victorian Department of Human Services, and Health Department of Western Australia. DD is supported by a Victorian Health Promotion Foundation public health research fellowship. OCU is supported by NHMRC Population Health Capacity Building Grant 436914 IS is supported by an NHMRC senior research fellowship. Competing interests: All authors have completed the Unified Competing Interest form at www.icmje.org/coi_disclosure.pdf (available on request from the corresponding author) and declare that all authors had: (1) No financial support for the submitted work from anyone other than their employer; (2) No financial relationships with commercial entities that might have an interest in the submitted work; (3) No spouses, partners, or children with relationships with commercial entities that might have an interest in the submitted work; (4) No Non-financial interests that may be relevant to the submitted work.

Ethical approval: The International Diabetes Institute Ethics Committee and the Human Research Ethics Committee of the University of Tasmania approved this study. All participants gave their consent by signing a consent form.

Data sharing: No additional data available.

1 Wilkin TJ, Voss LD. Metabolic syndrome: maladaptation to a modern world. / R Soc Med 2004:97:511-20.

2 Pan XR, Li GW, Hu YH, Wang JX, Yang WY, An ZX, et al. Effects of die and exercise in preventing NIDDM in people with impaired glucose tolerance: the Da Qing IGT and Diabetes Study. Diabetes Care 1997;20:537-44.

3 Tuomilehto J, Lindstrom J, Eriksson JG, Valle T, Hamalainen H, llanne-Parikka P, et al. Prevention of type 2 diabetes mellitus by changes in lifestyle among subjects with impaired glucose tolerance. N Engl J Med 2001;344:1343-50.

4 Knowler WC, Barrett-Connor E, Fowler SE, Hamman RF, Lachin IM, Walker EA, et al. Reduction in the incidence of type 2 diabetes with lifestyle intervention or metformin. N Engl J Med 2002;346:393-403.

5 Ramachandran A, Snehalatha C, Mary S, Mukesh B, Bhaskar AD, Vijay V. The Indian Diabetes Prevention Programme shows that lifestyle modification and metformin prevent type 2 diabetes in Asian Indian subjects with impaired glucose tolerance (IDPP-1). Diabetologia 2006;49:289-97.

6 Jeon CY, Lokken RP, Hu FB, van Dam RM. Physical activity of moderate intensity and risk of type 2 diabetes: a systematic review. Diabetes Care 2007:30:744-52.

7 Dwyer T, Hosmer D, Hosmer T, Venn AJ, Blizzard CL, Granger RH, et al. The inverse relationship between number of steps per day and obesity in a population-based sample: the AusDiab study. Int I Obes (Lond) 2007;31:797-804.

8 Bravata DM, Smith-Spangler C, Sundaram V, Gienger AL, Lin N, Lewis R, et al. Using pedometers to increase physical activity and improve health: a systematic review. JAMA 2007:298:2296-304.

9 Helmerhorst HJ, Wijndaele K, Brage S, Wareham NJ, Ekelund U. Objectively measured sedentary time may predict insulin resistance independent of moderate- and vigorous-intensity physical activity. Diabetes 2009;58:1776-9.

10 Ekelund U, Brage S, Griffin SJ, Wareham NJ. Objectively measured moderate- and vigorous-intensity physical activity but not sedentary time predicts insulin resistance in high-risk individuals. Diabetes Care 2009;32:1081-6.

11 Dunstan DW, Zimmet PZ, Welborn TA, Cameron Al, Shaw J, de Courten M, et al. The Australian Diabetes, Obesity and Lifestyle Study (AusDiab) - methods and response rates. Diabetes Res Clin Pract 2002;57:119-29.

12 Cameron AJ, Welborn TA, Zimmet PZ, Dunstan DW, Owen N, Salmon J, et al. Overweight and obesity in Australia: the 1999-2000 Australian Diabetes, Obesity and Lifestyle Study (AusDiab). Med J Aust 2003:178:427-32.

13 Australian Institute of Health and Welfare. The active Australia survey. AlHW, 2003.

14 Timperio A, Salmon J, Bull F, Rosenberg M. Validation of adult physical activity questions for use in Australian population surveys. University of Western Australia, 2002

15 Tudor-Locke C, Burkett L, Reis IP, Ainsworth BE, Macera CA, Wilson DK. How many days of pedometer monitoring predict weekly physical activity in adults? Prev Med 2005;40:293-8.

16 Ireland P, Jolley D, Giles G, O’Dea K, Powles J, Rutishauser I. Development of the Melbourne FFQ: a food frequency questionnaire for use in an Australian prospective study involving an ethnically diverse cohort. Asia Pacific J Clin Nutr 1994;3:19-31.

17 Australian Bureau of Statistics. Socio-economic indexes for areas (SEIFA), CD-ROM. ABS, 2001

18 Cameron AJ, Boyko EJ, Sicree RA, Zimmet PZ, Soderberg S, Alberti KG, et al. Central obesity as a precursor to the metabolic syndrome in the AusDiab study and Mauritius. Obesity (Silver Spring) 2008;16:2707-16

19 Magliano DJ, Barr EL, Zimmet PZ, Cameron AJ, Dunstan DW, Colagiuri S, et al. Glucose indices, health behaviors, and incidence of diabetes in Australia: the Australian Diabetes, Obesity and Lifestyle Study. Diabetes Care 2008;31:267-72.

20 Hall Al, Yee LI, Thomas SL. Life course epidemiology and infectious diseases. Int J Epidemiol 2002;31:300-1.

21 Wallace TM, Levy JC, Matthews DR. Use and abuse of HOMA modeling. Diabetes Care 2004:27:1487-95.

22 Royston P, Ambler G, Sauerbrei W. The use of fractional polynomials to model continuous risk variables in epidemiology. Int J Epidemiol 1999;28:964-74.

23 Little RJA, Rubin DB, eds. Statistical analysis with missing data. 2nd ed. Wiley, 2002 
24 Hatano Y. Use of the pedometer for promoting daily walking exercise. Int Council Health Phys Educ Rec 1993;29:4-8.

25 Marshall SJ, Levy SS, Tudor-Locke CE, Kolkhorst FW, Wooten KM, Ji M, et al. Translating physical activity recommendations into a pedometer-based step goal: 3000 steps in 30 minutes. Am J Prev Med 2009;36:410-5.

26 Tudor-Locke C, Ainsworth BE, Thompson RW, Matthews CE. Comparison of pedometer and accelerometer measures of free-living physical activity. Med Sci Sports Exerc 2002;34:2045-51.

27 Coke LA, Fletcher GF. Exercise and the cardiac patient-success is just steps away. J Cardiovasc Nurs 2010;25:238-40.
28 Samson MM, Crowe A, de Vreede PL, Dessens JA, Duursma SA, Verhaar HJ. Differences in gait parameters at a preferred walking speed in healthy subjects due to age, height and body weight. Aging (Milano) 2001;13:16-21.

29 Hellie T. How to measure your step or stride. 2010. www.bellaonline. com/articles/art39548.asp.

30 Ainsworth BE. The compendium of physical activities tracking guide. University of South Carolina, 2002. http://prevention.sph.sc.edu/ tools/docs/documents_compendium.pdf.

Accepted: 12 October 2010 